\title{
Landau Theory of the Finite Temperature Mott Transition
}

\author{
G. Kotliar*, E. Lange*, and M.J. Rozenberg ${ }^{+}$ \\ * Serin Physics Laboratory, Rutgers University, 136 Frelinghuysen Road, Piscataway, New Jersey 08854, USA \\ ${ }^{+}$Departamento de Física, FCEN, Universidad de Buenos Aires, Ciudad Universitaria Pab.I, (1428) Buenos Aires, Argentina.
}

(October 28, 2018)

\begin{abstract}
In the context of the dynamical mean-field theory of the Hubbard model, we identify microscopically an order parameter for the finite temperature Mott endpoint. We derive a Landau functional of the order parameter. We then use the order parameter theory to elucidate the singular behavior of various physical quantities which are experimentally accessible.
\end{abstract}

PACS Numbers: 71.30.+h, 71.10.Fd, 71.27.+a

When the strength of the electron-electron interaction $U$ is increased compared to the bare bandwidth $2 D$, a metal-insulator transition (MIT) occurs [1]. This phenomenon, known as the Mott transition, can take place in the absence of magnetic long-range order, and is still an outstanding problem in condensed-matter physics. From a theoretical point of view, a difficulty is the absence of an obvious order parameter to systematize the critical behavior of the observable quantities when the metal insulator transition is not accompanied by the onset of magnetic long range order. These issues are experimentally relevant to systems such as $\mathrm{V}_{2} \mathrm{O}_{3}$ and $\mathrm{Ni}(\mathrm{Se}, \mathrm{S})_{2}$ and are the subject of intensive experimental study. [2]

In recent years, great progress has been made by using the dynamical mean-field theory (DMFT) [3]. This framework describes both paramagnetic metallic and paramagnetic insulating phases. The $U-T$ phase diagram ( $T$ is the temperature) of the frustrated Hubbard model in the limit of large lattice coordination is qualitatively similar to that of the $\mathrm{V}_{2} \mathrm{O}_{3}$ and $\mathrm{Ni}(\mathrm{Se}, \mathrm{S})_{2}$ systems: A first-order phase-transition line ends in a second-order critical point, henceforth referred to as the Mott critical point, which is the main focus of this letter. We will use this framework to address the fundamental questions raised in the previous paragraph.

There are two earlier qualitative ideas as to what should be the order parameter to describe the physics around the finite temperature Mott point. One idea is to connect the order parameter to the notions of "metallicity" or coherence. It can be traced back to the early paper of Brinkman and Rice [4] and is captured in a slave boson formalism where the metallic state has a non zero expectation value of a Bose field which describes the coherent propagation of one particle excitations. [5] In a very different picture, Castellani et al. viewed the metal as a liquid rich in doubly occupied sites, and the insulator as a liquid with few doubly occupied sites. The metal to insulator transition is viewed as a condensation of doubly occupied sites, and the order parameter is related to the Blume-Emery-Griffith model [6]. The Landau approach presented here provides a synthesis of these ideas. It bridges naturally between a picture based on one par- ticle excitations and a picture based on local collective excitations (or double occupancies). In agreement with Castellani et al. we find that the Mott transition has indeed an Ising-like character. On the other hand, we obtain a complementary description in terms of the one particle spectral function reminiscent of the slave boson picture. A simple and clear description of the critical behavior near the critical point emerges. It allows us to systematically derive the critical behavior of any observable quantity and to relate its non analytic dependence on $T$ and $U$ to that of the order parameter. Our results should be also of help in resolving some controversies on the solution of the Hubbard model in infinite dimensions [7,8] by providing a theoretical framework in which to analyze numerical results on the finite temperature Mott transition. It can also be used to analyze results of photoemission and optical conductivity experiments.

For simplicity, we focus on the single-band Hubbard model at half-filling,

$$
\hat{H}=-\frac{t}{\sqrt{z}} \sum_{\langle i j\rangle \sigma} c_{i \sigma}^{+} c_{j \sigma}+U \sum_{i} \hat{n}_{i \uparrow} \hat{n}_{i \downarrow} .
$$

The first term describes the hopping between nearest neighbors on a lattice with coordination number $z$. The corresponding half bandwidth is our unit of energy, $D=2 t=1$. The second term is an on-site interaction suppressing double occupancies by imposing an energy cost $U$ on each one. In the limit of infinite dimensions, $z \rightarrow \infty$, this model can be mapped onto a single-impurity Anderson model (SIAM) supplemented by a self-consistency condition. We adopt a semicircular density of states, which is realized on the Bethe lattice. The dynamical mean-field equations can be obtained by differentiating the Landau functional

$$
F_{\mathrm{LG}}[\Delta]=-T \sum_{n} \frac{\Delta\left(i \omega_{n}\right)^{2}}{t^{2}}+F_{\mathrm{imp}}[\Delta]
$$

with respect to the hybridization function $\Delta\left(i \omega_{n}\right)$ of the SIAM, which has the meaning of a Weiss field. $i \omega_{n}$ are fermionic Matsubara frequencies, while $F_{\text {imp }}[\Delta]$ is the free energy of the SIAM, given by the action 
$S_{\mathrm{imp}}=S_{\mathrm{loc}}[\Delta=0]+\sum_{\sigma, n} f_{\sigma}^{+}\left(i \omega_{n}\right) \Delta\left(i \omega_{n}\right) f_{\sigma}\left(i \omega_{n}\right)$. Here, $S_{\text {loc }}[\Delta=0]$ is the action of the local $f$ level with the hybridization set to zero. The first term in Eq. (2) is the cost of forming the Weiss field $\Delta\left(i \omega_{n}\right)$ around a given site, while the second one is the free energy of an electron at this site in the presence of the Weiss field. Using the Green's function of the SIAM, $G\left(i \omega_{n}\right)=$ $(1 / 2 T) \delta F_{\mathrm{imp}} / \delta \Delta\left(i \omega_{n}\right)$, the mean-field equation reads

$$
\frac{t^{2}}{2 T} \frac{\delta F_{\mathrm{LG}}[\Delta]}{\delta \Delta\left(i \omega_{n}\right)}=t^{2} G\left(i \omega_{n}\right)[\Delta, \alpha]-\Delta\left(i \omega_{n}\right)=0 .
$$

Here, $\alpha=(U, T)$ comprises the control parameters. This Landau approach was used to describe the energetics of the Mott transition at zero temperature [9]. We will show that near the finite temperature Mott point, the Weiss field has a singular dependence which can be parametrized by a single number which assumes the role of an effective order parameter for this transition.

As in Landau theory, we assume that a finite temperature transition exists, and derive a complete description of the critical behavior near the transition as follows: First, we expand the mean-field equation (3) around the critical point, $\alpha_{c}=\left(U_{c}, T_{c}\right)$, up to third order in the deviation of the hybridization function from its value at the critical point, $\delta \Delta=\Delta\left(\alpha_{c}+\delta \alpha\right)-\Delta\left(\alpha_{c}\right)$, and to first order in $\delta \alpha=\left(U-U_{c}, T-T_{c}\right)$. This expansion is well-behaved because the impurity model at finite temperatures depends smoothly on $\alpha$ and $\delta \Delta\left(i \omega_{n}\right)$. In order to carry out this expansion it is convenient to define a fluctuation matrix

$$
M_{n m}=\left.\frac{t^{2}}{2 T} \frac{\delta^{2} F_{\mathrm{LG}}[\Delta]}{\delta \Delta\left(i \omega_{n}\right) \delta \Delta\left(i \omega_{m}\right)}\right|_{\text {critical point }}
$$

$M_{n m}$ has the form $-\delta_{n m}+K_{n m}$, where $K_{n m}$ is the Fourier transform of a kernel $K\left(\tau, \tau^{\prime}\right)$ which is proportional to the connected correlation function of an operator $O(\tau)=\int_{0}^{\beta} d u f^{+}(u+\tau) f(u),<O(\tau) O\left(\tau^{\prime}\right)>-<$ $O(\tau)><O\left(\tau^{\prime}\right)>$ where the average $<>$ is calculated with the action of an Anderson impurity model. It is well known that the correlation functions of the Anderson impurity model are bounded, and therefore the Kernel $K$ is square integrable $\int_{0}^{\beta} \int_{0}^{\beta} d \tau d \tau^{\prime}\left|K\left(\tau, \tau^{\prime}\right)\right|^{2}<\infty$. Therefore it $K_{n m}$ is a Fredholm operator which and has a discrete spectrum of eigenvalues which we labeled by the index $l$. 12]

At half-filling, particle-hole symmetry guarantees that the order parameter $\Delta(i \omega)$ is odd and wholly imaginary. Accordingly, the fluctuation matrix is real and symmetric and has real eigenvalues $m_{l}$ belonging to eigenvectors $\phi_{l}\left(i \omega_{n}\right)$ which can be chosen to be purely imaginary and to form an orthonormal basis. The critical point, in this description of the problem, is signaled by the appearance of a single zero eigenvalue, $m_{0}=0$, which indicates the occurrence of a simple bifurcation.
Next, we represent $\delta \Delta$ in the eigenbasis of the matrix (㺼), $\delta \Delta\left(i \omega_{n}\right)=\sum_{l} \eta_{l} \phi_{l}\left(i \omega_{n}\right)$, where all $\eta_{l}$ are real. By projecting the mean-field equation (3) onto the eigenbasis $\phi_{l}$, we obtain an equation of the form

$$
\begin{gathered}
m_{l} \eta_{l}+F_{l}^{(0)}\left[\left\{\eta_{j \neq 0}\right\}\right]+F_{l}^{(1)}\left[\left\{\eta_{j \neq 0}\right\}\right] \eta_{0} \\
+F_{l}^{(2)}\left[\left\{\eta_{j \neq 0}\right\}\right] \eta_{0}^{2}+F_{l}^{(3)} \eta_{0}^{3}=0,
\end{gathered}
$$

which holds for all $l . F_{l}^{(0)}$ is of order $\delta \alpha . F_{l}^{(1)}$ and $F_{l}^{(2)}$ have Taylor expansions in the $\eta_{j \neq 0}$, where $F_{l}^{(1)}$ starts with the linear order. We solve Eq. (5) iteratively for all $\eta_{l \neq 0}$ to obtain $\eta_{l \neq 0}=a_{l}+c_{l} \eta_{0}^{2}+d_{l} \eta_{0}^{3}$. Here, $a_{l}$ is of first order in $\delta \alpha$, (which assures us that the leading singular dependence of the spectral function is proportional to $\phi_{0}$ ) further corrections have the form $b_{l} \eta_{0}$ with $b_{l}$ also of order $\delta \alpha$. By inserting this expression into the $l=0$ case of Eq. (5), we derive an effective equation for the zero-mode amplitude $\eta_{0}$. We can think of $\eta_{0}$ as the soft mode near the transition and the $\eta_{l \neq 0}$ as massive modes. The elimination of the massive modes renormalizes the coefficients of the effective action for the soft mode. In the resulting cubic equation for $\eta_{0}$, we eliminate the quadratic term by shifting $\eta_{0}$ by an appropriately chosen linear function in $\delta \alpha, \eta=\eta_{0}+$ const $_{1} \times\left(T-T_{c}\right)+$ const $_{2} \times\left(U-U_{c}\right)$. Close to the critical point, $\eta$ and $\eta_{0}$ are dominated by non analytic terms and are therefore essentially equal. We thus obtain an equation of state without quadratic term in $\eta$ :

$$
p \eta+c \eta^{3}=h .
$$

Here, all quantities are real.

As in Landau theory, a microscopic calculation of the Landau coefficients $(\mathrm{p}, \mathrm{c}, \mathrm{h})$ is difficult. However we can extract exact information about the critical behavior from the knowledge that they are smooth functions of the control parameters, i.e. $c$ is finite at the critical point, whereas $p$ and $h$ are linear functions of $\delta \alpha, h=$ $h_{1}\left(U-U_{c}\right)+h_{2}\left(T-T_{c}\right)$ and $p=p_{1}\left(U-U_{c}\right)+p_{2}\left(T-T_{c}\right)$. As a consequence, $\eta$ has a singular dependence on $U$ and $T$ near the critical point. At $U=U_{c}$, and for $T$ near $T_{c}$,

$$
\eta\left(U_{c}, T\right) \simeq \operatorname{sign}\left(h_{2} / c\right) \operatorname{sign}\left(T-T_{c}\right)\left|T-T_{c}\right|^{1 / 3} .
$$

The mean-field equation (6) describes the Mott transition close to the critical point in terms of the order parameter $\eta$. In this form, the analogy with the liquid gas transition is evident. The Mott transition takes place on the line in the $U-T$ plane where $h$ vanishes and the system has full Ising symmetry. The critical point, $\left(U_{c}, T_{c}\right)$, divides this line into two half-lines. On the halfline where $T<T_{c}$, there are two solutions, $\eta= \pm \sqrt{|p / c|}$. We will see later that $\eta$ parametrizes the strength of the quasiparticle resonance of the single-particle spectrum (see Fig. 2). A positive or negative "field" $h$ increases or decreases this component of the spectral function, respectively. The field $h$ decreases when $U$ or $T$ is increased, 
because either increase eliminates the metallic coherence and thus reduces the value of $\eta$. We have used the sign convention whereby is positive.

We now turn to various consequences of our construction. From Eq. (6), we can obtain the shape of the coexistence region near the critical point, where two solutions of the mean field equations coexist. It is centered symmetrically about the $h=0$ line, and its width along $T=$ const lines, $\Delta U$, scales with $\left(T_{c}-T\right)^{3 / 2}$. The constant of proportionality is given by $\left(4 / \sqrt{c}\left|h_{1}\right|\right)\left[\left(p_{2}-p_{1} h_{2} / h_{1}\right) / 3\right]^{3 / 2}$.

An important quantity which is measured in numerical simulations is the double occupancy. It is connected to our order parameter $\eta$ as follows: $\langle d\rangle=$ $(T / U) \sum_{n}\left\{\left[\left(i \omega_{n}+\mu\right) G\left(i \omega_{n}\right)-1\right] e^{i \omega_{n} 0^{+}}-t^{2} G\left(i \omega_{n}\right)^{2}\right\}=$ $\langle d\rangle_{c}+c_{1}^{(d)} \eta+c_{2}^{(d)} \eta^{2}$. In this expansion about the critical point, we have only retained the leading and next to leading nonanalytic terms responsible for the critical behavior. The susceptibility $\chi=\partial\langle d\rangle / \partial U$ diverges at the critical point. For example:

$$
\chi\left(U, T_{c}\right) \simeq\left(c_{1}^{(d)} / 3\right) \operatorname{sign}\left(h_{1} / c\right)\left|h_{1} / c\right|^{1 / 3}\left|U-U_{c}\right|^{-2 / 3} .
$$

The double occupancy is related to the magnetization by the identity $\left\langle\left(n_{\uparrow}-n_{\downarrow}\right)^{2}\right\rangle=1-2\langle d\rangle$. The magnetic response will therefore also exhibit nonanalytic dependences on the control parameters.

There has been several numerical studies of the finite temperature Mott transition in this model. The Landau approach predicts the functional dependence of various quantities near the transition, and therefore the expressions derived in this paper, are useful for interpreting the numerical work. To illustrate how our approach sheds new light on previously obtained numerical data we compare in Fig. 1 the results for the double occupancy $\langle d\rangle$ obtained within the IPT and QMC calculations with $\Delta \tau=0.5 / D$, after carrying out the shifts and the rescaling described in the figure caption. Within the statistical errors of the QMC calculation, the agreement is excellent. This surprising result is consistent with the Landau theory: different approximations for the solution of the impurity model reduce to the same Landau theory near the critical point, but with different values of the Landau coefficients. Therefore, with a suitable rescaling, the results near the critical point should agree with each other, and with a fit based on the Landau theory which is shown in the red line in figure 1.

Small changes in the values of $\Delta \tau$ result in shifts of $U_{c}$, $T_{c}$, and $\langle d\rangle$ at criticality, but does not change the form of the critical behavior. We also note that the critical slowing down which has been observed in the iterative solutions of the mean field equations are a direct consequence of the presence of the soft mode $\eta$ described in the Landau approach.

From our construction it is clear that $\eta$ provides the leading non analytic behavior of the Weiss field. In order to get a better feeling for its physical significance

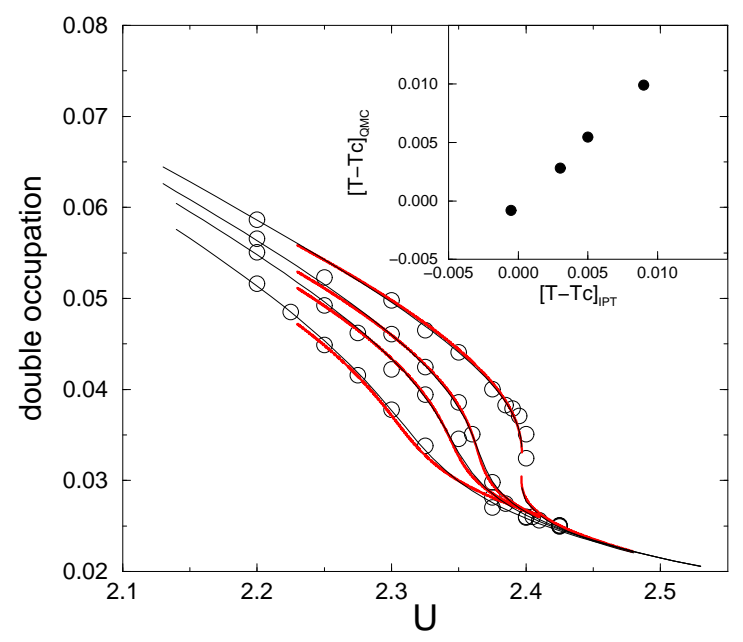

FIG. 1. Double occupation $\langle d\rangle$ as a function of $U$ for different temperatures. The thin black lines denote IPT results for $T_{\mathrm{IPT}}=0.0469,0.05,0.052,0.056$ (top to bottom). The thick red lines are a fit to the IPT data using the LG theory. The circles are QMC data obtained at $T_{\mathrm{QMC}}=1 / 40,1 / 35,1 / 32,1 / 25$ [ 8$]$. The IPT results where shifted by a constant -0.07 along the $U$ axis and by -0.003 along the $\langle d\rangle$ axis. The curves for the 3 larger temperatures are above $T_{c}$ and the lowest temperature ones (2 branches) are just below. The inset shows the scaling of the reduced temperatures $\left[T-T_{c}\right]_{\mathrm{QMC}}$ versus $\left[T-T_{c}\right]_{\mathrm{IPT}}$.

we have to understand how it can be probed experimentally. Since the order parameter is closely related to the amplitude of the quasiparticle peak, photoemission is an ideal tool to probe the temperature and pressure dependence of the order parameter near the critical point. This experimental technique, in the angle integrated mode, would also measure the convolution of the Fermi function with the analytically continued eigenfunction of the zero mode, $\operatorname{Im} \phi_{0}\left(i \omega_{n}=\omega-i \delta\right)$. To visualize the shape of the spectral function near the critical point we must resort to calculations based on analytic methods such as IPT. The inset of Fig. 2 shows the spectral function very near the critical point, computed within the IPT. It illustrates how the compromise between metallic and insulating features is realized. A finite $\eta$, depending on its sign, adds or subtracts spectral weight to the coherent low energy feature immersed in a constant backround in between the Hubbard bands. The zero mode is seen to affect mainly the low-energy part of the spectrum, which determines whether the system is metallic or insulating. The strong temperature dependence has been noticed in previous theoretical and experimental studies. [10] Its origin and connection to an order-parameter description of the Mott transition, however, had not been recognized until now. In the main panel of Fig. 2 2 we display the height of the quasiparticle peak $A_{0}=i \Delta\left(i 0^{+}\right) / \pi t^{2}$, for 


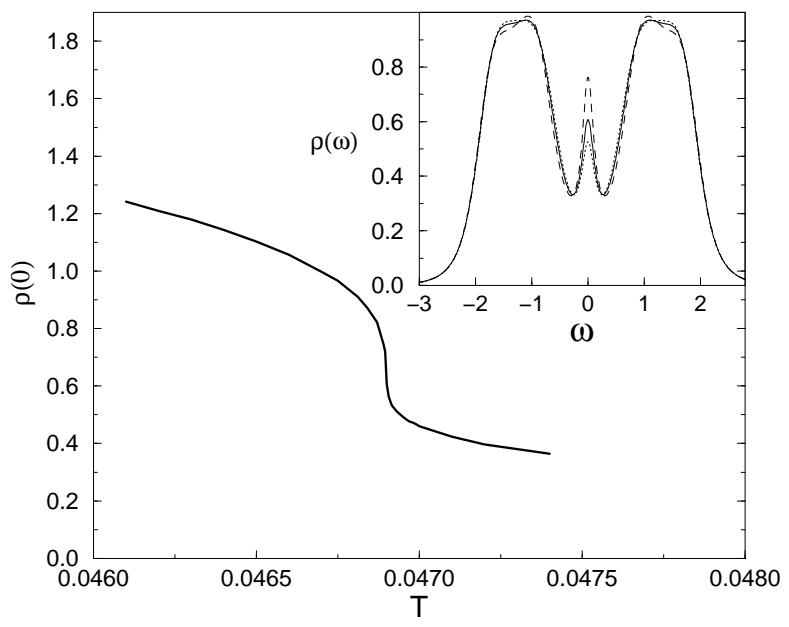

FIG. 2. The density of states at the Fermi energy $\rho(0) \equiv A_{0}$ as a function of temperature in the critical region $\left(U=2.46316 \approx U_{c}\right)$. The singular behavior of the slope at $T_{c} \approx 0.046897$ can be clearly appreciated. The inset shows the variation of the spectral function for $U \simeq U_{c}$ in the vecinity of $T_{c}$ : dashed line for $T-T_{c} / T_{c}=-0.00025$, solid line for $T-T_{c} / T_{c}=0.00006$ and dotted line for $T-T_{c} / T_{c}=0.00049$. 13

$U \simeq U_{c}$, as a function of temperature in the vicinity of $T_{c}$. The rapid variation seen in the figure is consistent with the form $A_{0}=A_{0 c}+c_{1}^{(A)} \eta+c_{2}^{(A)} \eta^{2}$ with coefficients $c_{i}^{(A)}$ independent of $\mathrm{U}$ and temperature.

Optical techniques are probably the best tool available to test the predictions of our theory. For instance, one may consider the integral of the optical conductivity up to some cuttoff, $N_{\text {eff }}(T)$. Since the optical conductivity in infinite dimensions is directly expressed in terms of the single-particle Green's function, $N_{\text {eff }}(T)$ must also exhibit the singular temperature dependence near the transition. We would therefore expect the temperature variation of this quantity to be most visible for a relatively small cuttoff, displaying a rapid variation with $T$ similarly as for $A_{0}$. Since the singular dependence arises from the order parameter $\eta$, it should be possible to fit the Drude weight by $N_{\text {eff }}(T)=N_{\text {eff }}\left(T_{c}\right)+$ $c_{1}^{(N)} \eta(T)+c_{2}^{(N)} \eta^{2}(T) . \quad N_{\text {eff }}(T)$ has recently been measured in $\mathrm{NiS}_{2-x} \mathrm{Se}_{x}$ [11, the observed strong temperature dependence of the effective number of carriers is consistent with our predictions.

In summary, we derived an order parameter description of the Mott transition near its critical point in the $U-T$ plane. We showed that the critical behavior in proximity to this point is governed by an Ising-like Landau functional and is present in a large number of observable quantities. We predict that any physical quantity which is sensitive to the single-particle spectrum exhibits singular dependences on the control parameters close to the finite-temperature Mott point. The leading non analytic behavior of other physical quantities can be obtained along similar lines, i.e. by recognizing their coupling to the order parameter. This involves a few coefficients, (i.e. the $c^{(A)}$ 's) which depend on the observable (and on the approximation method) and, as in Landau theory, should be taken as parameters. The dependence on temperature and on pressure is completely determined from the temperature or pressure dependence of the order parameter that follows from Eq. (6). ACKNOWLEDGMENT This work was supported by NSF 95-29138. E.L. was partially supported by the Deutsche Forschungsgemeinschaft. M.J.R. acknowledges support of Fundación Antorchas, CONICET (PID $N^{\circ} 4547 / 96$ ), and ANPCYT (PMT-PICT1855). We thank R. Chitra for discussions and D. Vollhardt for useful comments on the mansucript.

[1] N. F. Mott, Metal-Insulator Transitions (Taylor \& Francis, 1974).

[2] M. Imada, A. Fujimori, and Y. Tokura, Rev. Mod. Phys. 70, 1039 (1998).

[3] for a review see A. Georges, G. Kotliar, W. Krauth, and M. J. Rozenberg, Rev. Mod. Phys. 68, 13 (1996).

[4] W. F. Brinkman and T. M. Rice, Phys. Rev. B 2, 4302 (1970).

[5] G. Kotliar and A. E. Ruckenstein, Phys. Rev. Lett. 57, 1362

R. Fresard and P. Wolfle, Int. J. of Mod. Phys. B6, 685 (3087) (1986).

[6] C. Castellani, C. D. Castro, D. Feinberg, and J. Ranninger, Phys. Rev. Lett. 43, 1957 (1979).

[7] J. Schlipf, M. Jarrell, P.G.J. van Dongen, N. Blümer, S. Kehrein, Th. Pruschke, D. Vollhardt, Phys. Rev. Lett. 82, 4890 (1999).

[8] M. J. Rozenberg, R. Chitra, and G. Kotliar. Phys. Rev. Lett. 83, 3498 (1999). W. Krauth cond-mat/9908221

[9] G. Kotliar Eur. Jour. Phys. B, 11, 27-39 (1999).

[10] A. Y. Matsuura et al., Phys. Rev. B 53, 7584 (1996). Th. Pruschke D. Cox and M. Jarrell, Phys. Rev. B 47, 3553 (1993). A. Georges and W. Krauth Phys. Rev. B 48, 7167 (1993). M. Rozenberg et. al. Phys. Rev. Lett. 75, 105 (1995).

[11] S. Miyasaka and H. Takagi (unpublished).

[12] This is only true at finite temperatures, which ensures that the limit of integration $\beta$ is finite. At zero temperature the spectrum of fluctuations is continuos and the transition at a point denoted $U_{c 2}$ has a very different character and was described in Ref. [9].

[13] To accurately locate the critical point with IPT, we found it best to solve the DMFT equations on the imaginary axis. We note that closest to the $\left(U_{c}, T_{c}\right)$ point, up to 10,000 iterations were required to find converged solutions. 